\title{
VALOR DO SONO NATURAL OU INDUZIDO NO ELETRENCEFALO. GRAMA. RESLLTADO DE 500 OBSERVAÇÕES EM PACIENTES COM MOLÉSTIA CONVULSIVA
}

\author{
Jusé Geraldo Algernaz * \\ DORIS SAILER ALbernaZ **
}

Já em 1941, Gibbs e Gibbs "afirmavam que o sono constituía condição ideal para o estudo eletrencefalográfico da epilepsia. Outros trabalhos 2, 6, i, $8,9,11,12$ vieram demonstrar o crescente interêsse no estudo do sono aplicado à epilepsia. Não nos escapou o fato de que o uso de ativação cardiozólica, como vemos pelos trabalhos de Kaufman, Marshall e Walker ${ }^{10}$, de Cure, Rasmussem e Jasper "3, de Merlis, Henriksen e Grossman 13, de Cohn e Nardinj 1 e outros, ou fotocardiazólica, como preconiza Gastaut ${ }^{4}$, vêm também obtendo grande incremento.

Entretanto, o fato de estarmos instalados, não dentro de um hospital, como seria de desejar, mas em salas de edifícios no centro da cidade, não nos encorajou ao uso freqüente da ativação cardiazólica. Decidimos, pois, fazer um esfôrço no sentido de conseguir rotineiramente eletrencefalogramas pelo menos durante a vigília e o sono, empregando ainda a hiperventilação na quase totalidade dos casos e a ativação cardiazólica em alguns casos de interêsse especial.

O presente trabalho reflete nossa experiência inicial com eletrencefalogramas obtidos em consultório.

\section{METODO E MATERIAL}

O encefalógrafo de Grass, modêlo III-D, de 8 canais, foi usado em todos os casos. Os pacientes, recomendados para dormir pouco na véspera do exame, assentaram-se em confortável sofá-cama conversivel, colocado em cômodo especial, cujas paredes e características elétricas obedecem aos princípios da gaiola farádica. Foram usados rotineiramente 13 eletrodos no início de cada exame (dois frontais, dois centrais, dois occipitais, dois nas regiōes tem-

Trabalho apresentado ao II Congresso Sul-Americano de Eletrencefalografia e Neurofisiologia Clinica, Montevidéu, 21 a 24 março 1955.

* Docente da Clínica Neurológica da Fac. Med. da Univ. de Minas Gerais; diplomado pelo American Board of Neurological Surgery; Chefe do Serviço de Neurologia e Neurocirurgia do Hospital "Felicio Rocha" (Belo Horizonte, Minas Gerais).

** Técnica do Instifuto de Eletrencefalografia (Belo Horizonte, Minas Gerais). 
porais médias, dois nas temporais anteriores, dois nas orelhas e um no vértex). Outros eletrodos foram colocados conforme o indicassem a história do paciente ou as anormalidades encontradas no traçado. Ar condicionado, silêncio, escurecimento do cômodo, etc., constituíram ambiente propício à indução natural do sono. Em muitos pacientes, porém, ajudou-se o aparecimento do sono com o uso de Seconal, em doses que variaram de 0,02 a $0,20 \mathrm{~g}$ ( $0,05 \mathrm{~g}$ na maioria dos casos). Recentemente usamos Dormison em alguns casos.

De um total de 590 traçados, afastamos do presente estudo 82 por se referirem a pacientes sem história de moléstia convulsiva (encefalites, meningites, tumores, acidentes vasculares, neuroses, enxaqueca, etc.). Afastamos ainda 8 traçados de pacientes com história epiléptica, porque não foi conseguido traçado do sono. Restaram, pois, 500 eletrencefalogramas em vigília e sono, obtidos cronològicamente, em pacientes com história de moléstia convulsiva, com idade variando de 2 meses a 72 anos, e que constituem a base do presente estudo.

Releva notar que, em 590 eletrencefalogramas feitos no consultório, deixamos de obter sono apenas em 13 casos, ou $2,2 \%$, o que demonstra tratar-se de rotina perfeitamente exequível. Observamos ainda que, numa segunda tentativa, êsses pacientes poderiam vir a dormir durante o exame, como tivemos ocasião de verificar em alguns casos.

\section{RESULTADOS}

Obtivemos 129 traçados normais em vigilia e sono $(25,8 \%)$. Quanto aos demais: anormalidades surgiram apenas em vigilia em 110 casos $(22 \%)$; apenas em sono em $58(11,6 \%)$; em 203 casos $(40,6 \%$ ) foram demonstradas alterações tanto em vigília como em sono.

$\hat{E}$ importante observar que, neste último grupo, as anormalidades encontradas em sono coincidiam com as encontradas em vigília apenas em 73 casos. Nos outros 130, o eletrencefalograma durante o sono patenteou outros tipos de anormalidades. Em outras palavras, nesses 130 casos e nos 58 mencionados acima, totalizando 188 casos $(37,6 \%)$, o eletrencefalograma durante o sono mostrou anormalidades não reveladas pelo traçado de vigília.

Consideramos em nosso estudo todos os tipos de anormalidades eletrencefalográficas. Gibbs e Gibbs ${ }^{6}$, restringindo-se à observação de descargas convulsivas, encontraram, como era de esperar, uma grande diferença entre os resultados oferecidos pelos traçados de vigília e os de sono, o que evidencia ainda com mais clareza a vantagem do estudo eletrencefalográfico de epilépticos durante o sono.

Tipos de anormalidades encontradas - Cumulativamente, os tipos de anormalidades notados, respectivamente em vigilia (313 casos), em sono (261 casos), foram os seguintes: 


\begin{tabular}{|c|c|}
\hline & VIGÍLIA \\
\hline 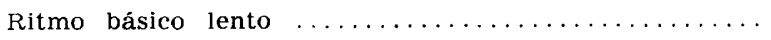 & 97 \\
\hline Ritmo básico rápido $\ldots \ldots \ldots \ldots \ldots \ldots \ldots \ldots \ldots \ldots$ & 31 \\
\hline Foco de onda ienta $\ldots \ldots \ldots \ldots \ldots$ & 21 \\
\hline Assimetria entre hemisférios $\ldots .$. & 94 \\
\hline 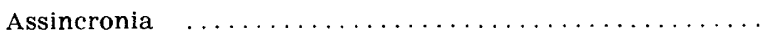 & 4 \\
\hline Focos espiculares, região temporal anterior $\ldots \ldots \ldots \ldots$ & 66 \\
\hline Focos espiculares, outras regiões $\ldots \ldots \ldots \ldots$ & 30 \\
\hline Espicula positiva a 14 e 6 por segundo $\ldots$ & 2 \\
\hline Espiculas múltiplas, generalizadas $\ldots \ldots$ & 6 \\
\hline Espicula-onda a 3 por segundo $\ldots \ldots \ldots \ldots \ldots \ldots$ & 15 \\
\hline Espicula-onda a 2 por segundo .... & 4 \\
\hline Espiculas múltiplas localizadas .. & 1 \\
\hline Ondas lentas paroxisticas $\ldots \ldots \ldots \ldots$ & 14 \\
\hline Variante de psicomotor $\ldots \ldots \ldots \ldots \ldots$ & - \\
\hline Miniatura de espicula-onda, a 6 por segundo. & - \\
\hline
\end{tabular}

\section{COMENTÁRIOS}

As anomalias paroxísticas surgiram durante as fases de sono com muito maior freqüência do que durante a vigília. O eletrencefalograma durante o sono foi altamente informativo, particularmente nos casos com focos temporais anteriores.

De 120 casos de focos temporais anteriores, em 43 o foco, unilateral, não foi sequer suspeitado durante a vigília, o mesmo acontecendo a mais 12 casos de focos bilaterais. Em mais 12 casos, nos quais foco espicular unilateral foi demonstrado em vigília, patenteou-se, durante o sono, a presença de focos bilaterais. Em todos os casos, menos um, focos demonstrados durante vigilia permaneceram durante as fases de sono, convindo assinalar que, às mais das vêzes, com maior nitidez.

As descargas de espículas positivas a 14 e 6 por segundo, correlacionadas por Gibbs e Gibbs ' como manifestações convulsivas de origem tálamo-hipotalâmica, surgiram apenas em dois casos durante a vigília e em 62 casos em sonolência ou sono; dêstes, 26 não apresentaram qualquer outra anormalidade, quer em vigilia, quer em sono.

Outras anormalidades paroxísticas reveladas em alguns casos durante o sono e não demonstradas pelo traçado de vigília incluem: descargas do complexo espicula-onda a 3 ciclos por segundo, ou a 2 ciclos por segundo, ou acompanhadas de espículas múltiplas; focos espiculares em localizações várias; descargas generalizadas de espiculas múltiplas; miniatura de espículaonda a 6 por segundo e ondas quadradas a 6 ciclos por segundo, principalmente nas regiões temporais ("variante de psicomotor").

O EEG durante o sono veio, em muitos dêsses casos, modificar grandemente o prognóstico e, em alguns, orientar decisivamente a conduta terapêutica. 


\section{RESUMO E CONCLUSOES}

1) Sono natural ou induzido com pequenas doses de Seconal ou Dormison por via enteral foi obtido em $97,8 \%$ dos pacientes submetidos à eletrencefalografia em consultório particular, ficando, pois, demonstrado tratar-se de rotina perfeitamente exequivel. 2) Em 11,6\% dos casos estudados o EEG durante o sono revelou anormalidades em pacientes com traçados de vigília inteiramente normais. 3) Em mais $26 \%$ dos casos patentou anormalidades completamente diferentes das observadas em vigília. 4) As anormalidades surgidas nos períodos de sono foram, em grande parte, de tipo paroxístico, revelando, com freqüência, modificações que se correlacionam com diferentes tipos clínicos de epilepsia e oferecem clementos de valor para prognóstico e tratamento. 5) O EEG durante o sono foi particularmente útil no estudo dos focos espiculares das regiões temporais anteriores $\_$na demonstração das descargas de origem tálamo-hipotalâmica descritas por Gibbs e Gibbs ‘. 6) Em nossa opinião, sono não deve ser considerado "método de ativação" para ser usado em casos com traçados de vigília normais, mas deve fazer parte do exame eletrencefalográfico de rotina, sem o que o EEG deve ser considerado incompleto.

\section{SUMMARY AND CONCLUSIONS}

\section{Value of natural or induced sleep in electroencephalography}

1) Natural or induced sleep was obtained in 97.8 per cent of the patients submitted to electroencephalography in the office, showing therefore, that sleep may be used routinely with ease. 2) In 11.6 per cent of the cases sleep records showed abnormalities in patients with entirely normal wake records. 3) In 26 per cent of the cases demonstrated abnormalities which were entirely different from those seen in the make records. 4) Most abnormalities appearing during sleep were paroxysmal in type and frequently demonstrated patterns which correlate well with different clinical types of epilepsy and offered valuables clues to prognosis and treatment. 5) During sleep, the E.E.G. was particularly useful in the study of spike-focus in the anterior temporal regions and in demonstrating discharges of thalamo-hypothalamic origin. 6) In our opinion, sleep is not to be considered an "activation technique" to be used in otherwise normal tracings, but should be done routinely in order to consider the E.E.G. complete.

\section{BIBLIOGRAFIA}

1. COHN, R.; NARDINI, J. E. - Spike-dome discharges in selected psychiatric patients activated by I.V. Metrazol. American EEG Society, New Jersey, 1951. 2. COHN, R.; NARDINI, J. E.; BOSWELL, W. E. - A comparison of provocative agents in the epilepsies and in controls. Neurology, 2:481-487, 1952. 3. CURE, C.; RASMUSSEN, T.; JASPER, H. - Activation of seizures and electroencephalographic disturbances in epileptics and in control subjects with Metrazol. Arch. Neurol. a. Psychiat., 59:691-717, 1948. 4. GASTAUT, H. - Combined photic and Metrazol activation of the brain. EEG Clin. Neurophysiol., 2:249-260, 1950. 5. GIBBS, F. A.; GIBBS, E. L. - Atlas of Electro- 
encephalography. Lew A. Cummings Co., Cambridge (Mass. U.S.A.), 1941. 6. GIBBS, E. L.; GIBBS, F. A. - Diagnostic and localizing value of electroencephalographic studies in sleep. Ass. Research Nerv. a. Ment. Dis. Proc,, 26:366-367, 1946. 7. GIBBS, F. A.; GIBBS, E. L. - Atlas of Electroencephalography, ed. 2. Adidson-Welwy, Cambridge (Mass. U.S.A.), 1950. 8. GIBBS, E. L.; GIBBS, F. A.; FUSTER, B. - Psychomotor epilepsy. Arch. Neurol. a. Psychiat., 60:331-339, 1958. 9. GROSSMAN, C.; GOLUB, L. M.; MERLIS, J. K. -- Influence of sleep on various forms of abnormality in the electroencephalogram. EEG Clin. Neurophysiol., 4:195-200, 1952.10 . KAUFMAN, I. C.; MARSHALL, C.; WALKER, A. E. - Metrazol activated electroencephalography. Ass. Research Nerv. a. Ment. Dis. Proc., 26:476-486, 1957. 11. LI, C. L.; JASPER, H.; HENDERSON, L. - The effect of arousal mechanism on various forms of abnormality in the electroencephalogram. EEG Clin. Neurophysiol., 4:513-526, 1952. 12. MERLIS, J. K.; GROSSMAN, C.; HENRICKSEN, G. F. - Comparative effectiveness of sleep and Metrazol activated electroencephalography. EEG Clin. Neurophysiol., 3:71-78, 1951.13. MERLIS, J. K.; HENRICKSEN, G. F.; GROSSIMAN, C. - Metrazol activations of seizure discharges in epileptics with normal routine electroencephalograms. EEG Clin. Neurophysiol., 2:17-22, 1950.

Rua Carijos, $141-$ Belo Horizonte, Minas Gerais - Brasil. 